OPEN ACCESS

Edited by: Henk F. Moed, Sapienza University of Rome,

Reviewed by:

Saeed-Ul Hassan, Information Technology University,

Pakistan

Thed Van Leeuwen, Leiden University, Netherlands

${ }^{*}$ Correspondence:

Ping Zhou

pingzhou@zju.edu.cn

Received: 05 February 2016 Accepted: 07 July 2016 Published: 02 August 2016

Citation:

Zhou P and Leydesdorff L (2016)

A Comparative Study of the Citation

Impact of Chinese Journals with Government Priority Support.

Front. Res. Metr. Anal. 1:3. doi: 10.3389/frma.2016.00003

\section{A Comparative Study of the Citation Impact of Chinese Journals with Government Priority Support}

\author{
Ping Zhou ${ }^{1 *}$ and Loet Leydesdorff ${ }^{2}$ \\ ${ }^{1}$ Department of Information Resources Management, School of Public Affairs, Zhejiang University, Hangzhou, China, \\ ${ }^{2}$ Amsterdam School of Communication Research (ASCoR), University of Amsterdam, Amsterdam, Netherlands
}

In order to improve the citation impact of Chinese journals, relevant Chinese government agencies have launched a program entitled Citation Impact Upgrading Plan (CIUP) with financial support. Only a few journals that perform better have been screened out for CIUP support. This study tries to figure out if the screening results reflect the bibliometric status of the journals. We compare journals being supported by CIUP with unsupported ones. Journals supported by the CIUP have made progress in raising their journal impact factor (JIF) values and perform better in terms of JIF values. Journals citing CIUP journals have a spectrum of higher JIF values. Journals in a cited network of a CIUP journal are better integrated with one another. However, wide gaps still exist between Chinese and leading international journals. A network visualization of being-cited patterns can be used to measure a journal's citation impact in relation to the JIF values of journals in the network. Policy suggestions on improving citation impact of Chinese journals will be proposed.

Keywords: Chinese journal, citation impact, cited network, international visibility, government support

\section{INTRODUCTION}

Right after the United States, China has been the second largest producer of scientific publications since 2006 (Zhou and Leydesdorff, 2008; ISTIC, 2013). With citation impacts rising continuously, China jumped to the fifth position in 2013 in terms of national total citations from the eighth in 2010 (ISTIC, 2013). This position was reached 2 years earlier than targeted by the Ministry of Science and Technology (MOST) of China in the 12th National Plan for the Development of Science and Technology (NPDST). In terms of total citations received by journals in specific disciplines, however, significant variation exists with journals in chemistry, materials science, engineering technology, mathematics, computer science, and physics receiving the most citations, and thus taking the second position in the shares of total world (ISTIC, 2013).

In addition to being a second largest producer of academic papers, China is also the second largest publishing nation of academic journals. Of the 9,884 Chinese journals, approximately 5,300 are in science and technology (Liu, 2012; Yao et al., 2014). International visibility of Chinese journals is still low but improving (Jin and Rousseau, 2004; Leydesdorff and Jin, 2005; Zhou and Leydesdorff, 2007a,b; ISTIC, 2013): in addition to a growing number of journals being included in Science Citation Index - Expanded (SCIE), the number of journals in the first and second quartiles of the journal impact factors (JIFs) have also increased. 
In 2014, 173 Chinese journals were included in the Journal Citation Reports (JCR), of which 22 (12.5\%) in the first quartile and $42(23.9 \%)$ in the second quartile, whereas the corresponding figures were, respectively, 160, 11 (6.9\%), 40 (25.0\%) in 2013. ${ }^{1}$ The number of journals in the first quartile doubled within 1 year. Nevertheless, international visibility of Chinese journals is still low in terms of both inclusion ratios in WoS and JIF values: only around $3 \%$ of China's 5,300 S\&T journals are indexed in the SCIE, and JIF values of most of these journals (63.6\% in 2014) are in the lowest (third and fourth) quartiles of the JCR (see text footnote 1).

In order to raise international visibility and especially the citation impact of Chinese journals, relevant government agencies of China have launched a series of supporting projects. Different government agencies provide incentives with different objectives. Before moving further, it is necessary to explain the complex administrative structure of Chinese journals (Figure 1). At the national level, administration is carried out by State Administration of Press, Publication, Radio, Film and Television (state APPRFT) after integration with other government agencies. The APPRFT is under the direct control of the State Council of China and has branches at each province and municipality. In addition to making regulations and policies relevant to journal publishing and development, State APPRFT has the power of authorizing establishment of new journals and carries out routine censorship, whereas provincial APPRFTs are responsible for administration and control (including censorship) of local journals.

Routine management of Chinese academic journals is carried out by editorial boards affiliated with research institutes, universities, and academic associations/societies. The institutions are also affiliated with respective government agencies. Different governmental agencies are responsible for different sets of journals with different policies aiming at quality improvement, but with a common focus on international visibility. For

${ }^{1}$ https://jcr.incites.thomsonreuters.com/JCRJournalHomeActionaction?SID=A2uBT1Q0j5w0vJ6KAtJkx2BYatx2BlbTBnOasF-18x2dDERVkILV0BdTPTpUU0 DGKQx3Dx3DmUK7LrcuxxAJgHcx2FVlEhO0wx3Dx3D-9vvmzcndpRgQC GPd1c2qPQx3Dx3D-wx2BJQh9GKVmtdJw3700KssQx3Dx3D\&SrcApp=IC2LS \&Init=Yes

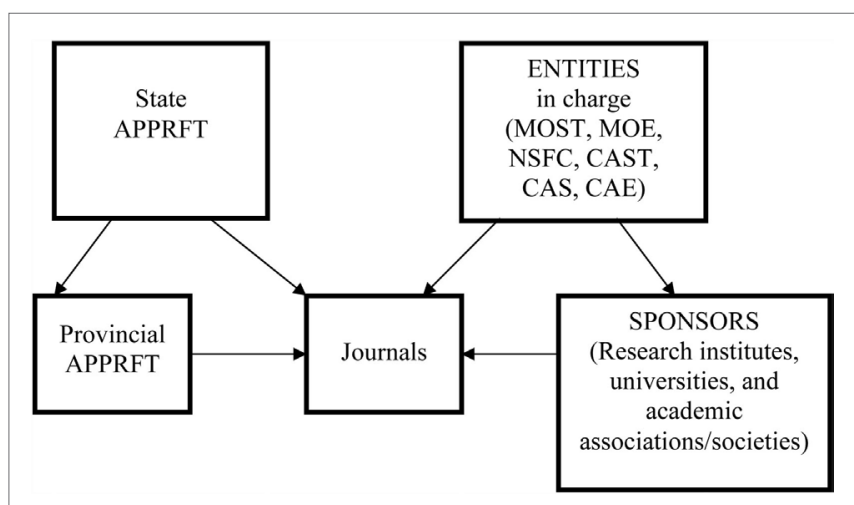

FIGURE 1 | The administrative structure of Chinese journals. example, national-level projects include the Journal Phalanx of China of State APPRFT, the Development Strategy Research for Competitive S\&T Journals of the MOST, and the Key Academic Specific Foundation of the National Natural Science Foundation of China (NSFC). Years have passed since the launch of the above projects, and the original targets of raising journal impact and international visibility have remained beyond reach.

In order to improve the situation, another financial support project - the International Impact Upgrading Plan for Chinese S\&T Journals (abbreviated as CIUP) - was jointly launched, in November 2013, by six government agencies, including the State APPRFT, the China Association for Science and Technology (CAST), the Ministry of Finance, the Ministry of Education (MOE), the Chinese Academy of Sciences (CAS), and the Chinese Academy of Engineering (CAE). The CIUP is carried out in two steps. The first step was to raise JIF values of a selected set of journals to quartiles 1 and 2 in JCR by the end of the 12th 5-Year Plan (2011-2015) and to establish a journal set that can represent research frontiers or dominant fields of China, or in fields in which China does not have its own journals yet. The second step is to form a world-top-journal set in which China has independent intellectual property rights by the year 2020 .

Candidate journals for CIUP support must be published in English and under the management of one of the above six government agencies. To ensure high-quality journals to be supported, the selection scheme combines bibliometric indicators, expert reviews, and an assessment of editorial boards. In total, 76 S\&T journals have been selected for CIUP support. Compared with the 5,300 scholarly journals in science and technology, the 76 supported journals may seem few, but these are almost half (47\%) of the Chinese journals indexed in the SCIE in 2013.

The supported journals are classified into four categories, namely $\mathrm{A}, \mathrm{B}, \mathrm{C}$, and $\mathrm{D}$, and receive annual support of 2 million RMB (322,092 US\$), 1 million RMB (161,046 US\$), 0.5 million RMB (85,230 US\$), and only once with 0.5 million $\operatorname{RMB}(85,230$ US\$), respectively. Basic conditions for journals in categories A, $\mathrm{B}$, and $\mathrm{C}$ are two - published in English and indexed in SCIE or Environment International (EI) or MedLine. Journals in category $\mathrm{D}$ are not required to be in English but will have an English version in the future. Financial support for journals in category D last for 3 years, and 10 journals are selected each year. The number of journals supported by the CIUP is $6,30,30$, and 10 in categories A, B, C, and D, respectively. Journals in category A are all indexed in the SCIE, whereas some in the other three categories (i.e., B, C, and D), however, are not included in SCIE in 2013.

In this paper, we investigate if the CIUP scheme corresponds with citation impact in terms of cited patterns of a target JIF and country of origin of journals in its cited environment. Is a journal in a higher rank of a supporting category more influential internationally or domestically (i.e., cited by more international or Chinese journals) or cited by more journals with higher JIF values? Does disciplinary/field variation exist in the above issues? The investigation will be done by comparing between CIUP supported and unsupported Chinese S\&T journals indexed in the SCIE. Furthermore, cited patterns of leading international journals in the same subject category of the JCR will also be included 
in the comparison so as to figure out the gap between Chinese and leading international journals.

In fact, comparative studies between Chinese and international journals have been carried out before (Jin and Leydesdorff, 2005; Li, 2006; Zhou and Leydesdorff, 2007a,b; Zhou et al., 2010). For example, based on data of the JCR of Thomson Reuters and the China Scientific and Technical Papers and Citations Database (CSTPCD) of the Institute of Scientific and Technological Information of China (ISTIC; Zhou and Leydesdorff, 2007a,b), compared journal-journal citation relations from different perspectives, and found that international visibility of highquality Chinese journals was low. Years have passed since the previous studies, and the situation has changed with China's rapid development in science and technology and its increasing R\&D investment during the past 10 years (MOST, 2012; NBS, 2013). The launch of CIUP ignites our interests of mapping an updated picture of Chinese journals by focusing on the supported versus unsupported Chinese journals in terms of their respective positions in the international scholarly community and to assess the rationality of CIUP in selecting journals of better performance in the international community.

\section{MATERIALS AND METHODS}

We apply routines developed by Leydesdorff and Cozzens (1993) with aggregated journal-journal citation matrices are harvested from JCR data from the perspective of a seed journal. A seed journal is the one under investigation and acts as a starter to run the routines. Any journal indexed in Science Citation Index (SCI) or Social Science Citation (SSCI) can be used as a seed. The resulting ego-network is composed of journals citing or cited by a seed journal. Journals citing a seed journal form a citing network and those cited by a seed journal constitute a cited network. A citation network includes all journals that cite or are cited by a seed journal to the extent of a contribution of, for example, $1 \%$ of the seed journal's total citation rate (He and Pao, 1986; Leydesdorff, 1986). By default, the threshold is $1 \%$ in the routines, but this can be changed so as to include an appropriate number of journals in a local citation network. For a network with too many journals, one can raise the threshold in order to reduce the size of the network, and vice versa.

Each journal in a network is represented by a node which can be a circle or an ellipse in a Pajek picture. ${ }^{2}$ The size of an ellipse is determined by the corresponding journal's contribution to the citing or cited environment in the year under investigation. The distinction of the vertical and horizontal size of the ellipse informs the reader about the extent to which within-journal (self-)citations participate in the citation impact (Leydesdorff, 2007; Zhou and Leydesdorff, 2008). Note that within-journal citations can be author self-citations or citations among authors publishing in the same journal. Citations excluding journal self-citations can be considered as a measure of inter-journal communication.

${ }^{2}$ Pajek is a routine for the analysis and visualization of large networks, freely available at http://mrvar.fdv.uni-lj.si/pajek/
In a citation environment, a journal's node size is determined by the logarithm of its contribution to the total number of citations in a local environment during the year under investigation. Citation counts are based on total citations to a journal during the current year and are combined for both the SCI and SSCI.

Many programs, such as VOSviewer, Pajek, or Gephi, can be used to visualize journal citation networks. In this study, we use Pajek because it serves the purpose of illustrating the relative cited size of individual journals in local environments. Data of a citation environment can be imported into Pajek after being generated by the routines. The cosine between two vectors (Salton and McGill, 1983 ) is used to measure the similarity between the distributions of various journals included in a citation network (Leydesdorff, 2007). A visualized citation network showing strength of citation relations between journals in a local environment can thus be obtained.

To display citation impact of a seed journal, cited relations are generated from the 2013 version of the JCR of Thomson Reuters. Comparison is carried out between three types of journals, namely supported and unsupported Chinese journals, as well as leading international journals. Cited counts of a journal are all citations to items published in the journal in the past 10 years of 2013 including the year 2013. Such calculation may result in a bias to journals included in the SCIE in different years: a journal indexed earlier may have more chances of being cited in the current year. To avoid or reduce this possible bias, we select journals indexed in the same year or journals at least 10 years (i.e., in or before 2004) in the SCIE in the same subject category.

To select journals supported by the CIUP, two criteria are applied. First, a journal must be listed in categories A or B of the CIUP, because journals in these two categories are supposed of relatively higher quality than those in categories $\mathrm{C}$ and $\mathrm{D}$ according to the support conditions of CIUP. Second, a journal must have been indexed in SCIE for at least 10 years (Slyder et al., 2011; Finardi, 2014) so as to ensure an equal or at least similar length of the citation window. To investigate possible disciplinary/field variation, journals in different disciplines/fields are included for comparative analysis. Journals with title changes were not included because of a possible discontinuity of citation data. For example, under the title Science in China, there were series A to E representing different fields until 2011 and then the journal titles were changed to Science China plus field names such as Science China-Mathematics, Science China-Chemistry, and so on. The citation data of such title-changed journals in JCR have not been fully integrated yet.

Based on the above criteria, a few journals in categories A and $\mathrm{B}$ of CIUP can be selected (Table 1). The EI and Communications on Pure and Applied Mathematics (CPAM) were added for illustrating the gap between Chinese and leading international journals.

The citation impact of a seed journal is positively related to its JIF and also JIFs of journals in the seed journal's cited network. In addition, the academic reputation of the editor and editorial board members plays a role. Editorial board members of leading international journals are from all over the world with high academic impact, whereas those of Chinese journals are less so. Therefore, assessing international visibility should also consider 
TABLE 1 | Journals being analyzed.

\begin{tabular}{|c|c|c|c|c|c|c|c|c|c|}
\hline \multirow{2}{*}{$\begin{array}{l}\text { Subject } \\
\text { category }\end{array}$} & \multirow[t]{2}{*}{ Journal title } & \multicolumn{4}{|c|}{ JIF } & \multirow{2}{*}{$\begin{array}{l}\text { All year } \\
\text { citations }\end{array}$} & \multirow[t]{2}{*}{ CIUP category } & \multirow{2}{*}{$\begin{array}{l}\text { Initial year } \\
\text { in JCR }\end{array}$} & \multirow{2}{*}{$\begin{array}{l}\text { Country } \\
\text { origin }\end{array}$} \\
\hline & & 2008 & 2013 & Growth & Growth (\%) & & & & \\
\hline \multirow[t]{3}{*}{$\begin{array}{l}\text { Environmental } \\
\text { Sciences }\end{array}$} & $\begin{array}{l}\text { Journal of Environmental Sciences - China } \\
\text { (JESC) }\end{array}$ & 0.720 & 1.922 & 1.202 & 166.9 & 4,774 & A & 2003 & China \\
\hline & Biomedical and Environmental Sciences (BES) & 0.675 & 1.257 & 0.582 & 86.2 & 1,343 & Not applicable & 1998 & \\
\hline & Environment International (El) & 3.516 & 5.664 & 2.148 & 61.1 & 10,171 & Not applicable & 1997 & USA \\
\hline \multirow[t]{3}{*}{ Mathematics } & Journal of Computational Mathematics (JCM) & 0.765 & 1.049 & 0.284 & 37.1 & 815 & $\mathrm{~B}$ & 1997 & China \\
\hline & Acta Mathematica Scientia (AMS) & 0.222 & 0.620 & 0.398 & 179 & 593 & Not applicable & 1997 & \\
\hline & $\begin{array}{l}\text { Communications on Pure and Applied } \\
\text { Mathematics (CPAM) }\end{array}$ & 3.806 & 3.080 & -0.726 & -19.1 & 6,904 & Not applicable & 1997 & USA \\
\hline
\end{tabular}

the country of origin of journals citing a seed journal, in addition to JIF values. In a cited network, the more journals from foreign countries, the higher the international visibility a journal is.

In a visualized cited network, a journal is considered as well integrated into the scholarly communication of a specific field if most journals in the network cite one another. With limited time and specific research interests, authors, especially those publishing in high-impact journals, may not read all publications in their fields. Instead, they focus on publications in journals of high-impact and/or of specific interests, which may result in stable citations to high-impact journals. Furthermore, authors being able to publish in high-impact journals are more likely to cite papers in high-impact journals, and thus resulting in a wellintegrated citation network. Journals with fewer citation relations and at the edge of a citation network are less frequently accessed by scholars in a specific scholarly community, and thus have fewer chances of being cited. High-impact journals in a specific field are more likely to generate a well-integrated citation network, whereas multidisciplinary journals and journals of low impact are less likely to have an integrated citation network because of being less frequently accessed by scholars.

\section{RESULTS}

The study focuses on the cited patterns of the selected journals in 2013. When visualizing a cited network of a seed journal, the threshold was set at $1 \%$. In other words, journals appearing in a cited network contribute at least $1 \%$ to the total citations of a seed journal. The maps are based on cosine $\geq 0.2$.

\section{Citation Impact of Journals in Environmental Sciences}

In environmental sciences, Journal of Environmental Sciences - China (JESC) is supported under category A by the CIUP, whereas Biomedical and Environmental Sciences (BES) is not. In 5 years from 2008 to 2013, JIF of JESC has increased by $166.9 \%$ from 0.720 to 1.922 , and that of BES has also increased but with a lower ratio (86.2\%) from 0.675 to 1.257 (Table 1). JESC outperforms BES in terms of both absolute value and growth rate of JIF, resulting in an enlarged gap between the two journals because of increased JIF difference from 0.045 (in 2008) to 0.565 (in 2013). The number of journals contributing more than $1 \%$ of the total citations of JESC is significantly more than that of journals citing BES, which may imply a wider scope of impact of JESC.

Journal impact factor values of journals contributing at least $1 \%$ of the total citations of JESC range from 0.527 of Fresenius Environmental Bulletin to 5.323 of Water Research, and JIFs of those citing BES range from 1.257 of BES to 3.534 of Plos One. In other words, JESC has impact on journals with much higher JIF values. In terms of being cited by foreign journals, both JESC and BES receive citations from foreign journals (Figures 2A,B). Nevertheless, international visibility of JESC is clearly higher than that of BES because of significantly more journals citing JESC.

Regarding structure of cited networks (Figure 2A), JESC is surrounded by journals in environmental sciences except Plos One - a multidisciplinary journal. Most journals cite one another in addition to citing JESC. Nevertheless, holes exist in the network. Journals, such as Fresenius Environmental Bulletin, Desalination and Water Treatment, and Ecological Engineering, do not link with other journals in the network. In other words, not all journals in the cited network of JESC have established citation relations with each other.

In terms of integration in its own network (Figure 2B), BES performs worse: the only four journals in its cited network do not cite each other. This situation may lead to unstable citation relations of BES, and thus affect the increase of citation impact (e.g., JIF value). Compared with journals in the cited network of JESC, such as Water Research, Journal of Hazardous Materials, and Chemosphere, JESC has received much fewer citations, but the reception of BES is even worse.

With already much higher JIF (3.516) value than JESC (0.720) in 2008, the Environment International (EI) outperforms JESC in raising JIF value, which widens the gap between the two. This implies a harder task for JESC to catch up with leading international journals such as EI. Scope of citation impact of $E I$ is wider with 15 journals contributing at least $1 \%$ to its total citations. JIF values of journals in the cited network of EI range from 1.679 of Environmental Monitoring and Assessment to 5.323 of Water Research. Average JIF value (3.607) of journals citing EI is higher than that of JESC (2.941). Journals except Plos One in the cited network of $E I$ are completely integrated with each other (Figure 2C). In other words, journals in the cited network of EI integrate better than those in the two Chinese journals. 


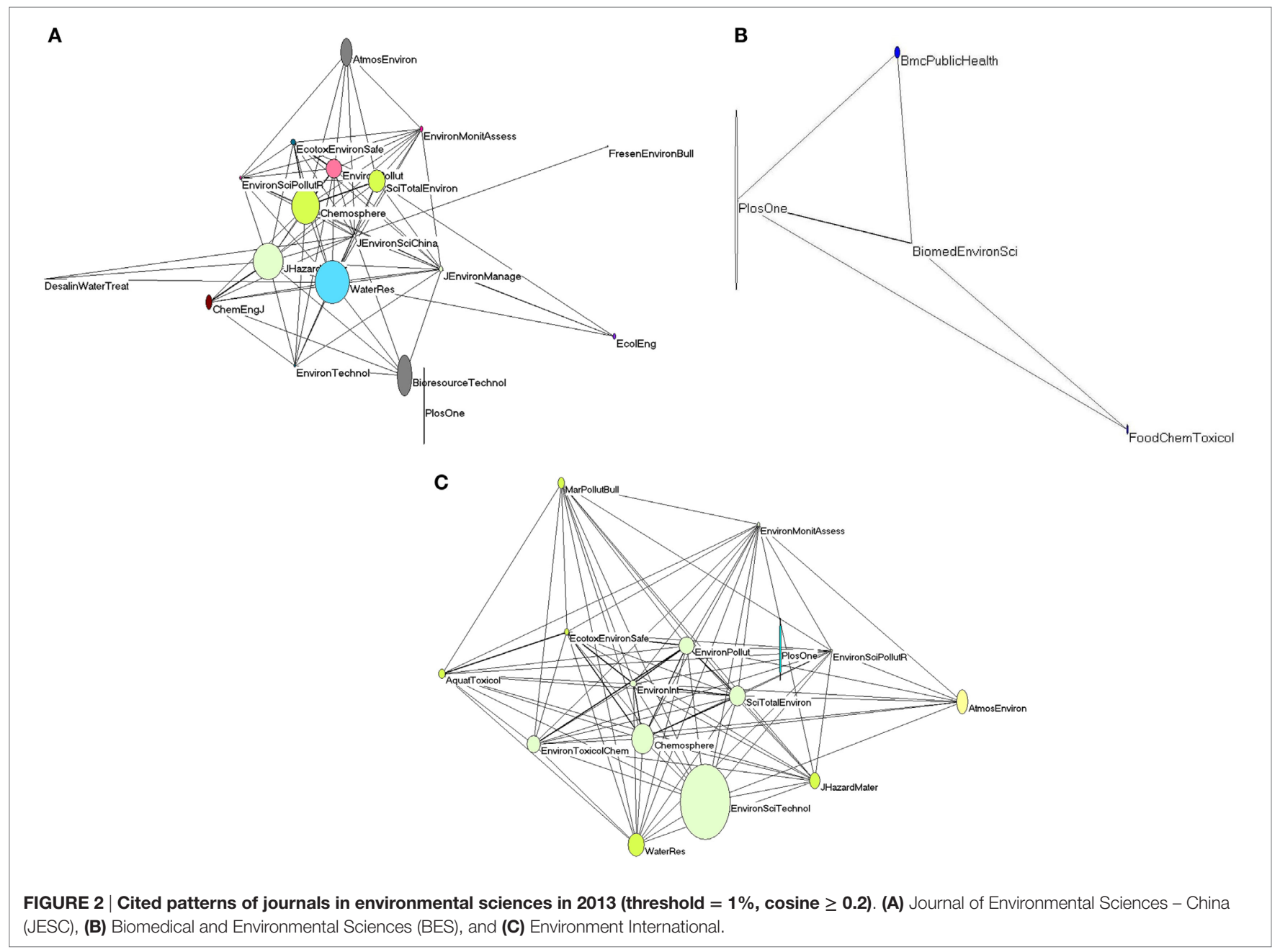

\section{Citation Impact of Journals in Mathematics}

Of the three journals in mathematics in Table 1, Journal of Computational Mathematics (JCM) and Acta Mathematica Scientia (AMS) are from China and CPAM is from USA. JCM is supported under category B of CIUP, whereas AMS in not supported by the CIUP. Both journals are under the subject category of mathematics and have been indexed in SCIE since 1997. CPAM is a top journal in mathematics in terms of JIF value and is selected for international comparison for the two Chinese journals.

Journal of Computational Mathematics performs better than AMS in absolute JIF value, but not in relative growth rate. The JIF value of JCM has been increased by $37.1 \%$ from 0.765 to 1.049 in the 5 years, whereas that of AMS increased by $179.0 \%$ from 0.222 to 0.620 . JIF difference between the two journals has been reduced slightly from 0.543 to 0.429 (Table 1). By 2013, the cited networks of JCM and AMS cover, respectively, 25 and 16 journals, which implies a wider impact scope of JCM compared with that of AMS.

The JIF values of journals in the cited network of JCM range from 0.673 of International Journal of Numerical Analysis and
Modelling to 2.485 of Journal of Computational Physics, whereas those in the cited network of AMS range from 0.620 of AMS to 2.486 of Fixed Point Theory and Applications. Thus, the two journals have similar impact on a journal set of similar JIF values. With most citing journals originated from foreign countries, the international visibilities of both journals are similar (Figures 3A,B).

In its cited network (Figure $\mathbf{3 A}$ ), JCM is surrounded by journals in mathematics, indicating a good integration of JCM in the scholarly community of mathematics. In fact, all journals in the cited network of JCM are in mathematics and mostly link (cite) each other forming an integrated unity. Nevertheless, some journals, such as Linear Algebra and Applications, Science China-Mathematics, Computer Physics Communications, and Mathematical Problems in Engineering, do not have links with other journals in the network.

Located at the edge of its own cited network (Figure 3B), AMS is less integrated into the cited network compared with JCM. In addition to Scientific World Journal which is completely isolated from the network, journals, such as Frontiers in Mathematics in China, Fixed Point Theory and Applications, and Journal of 

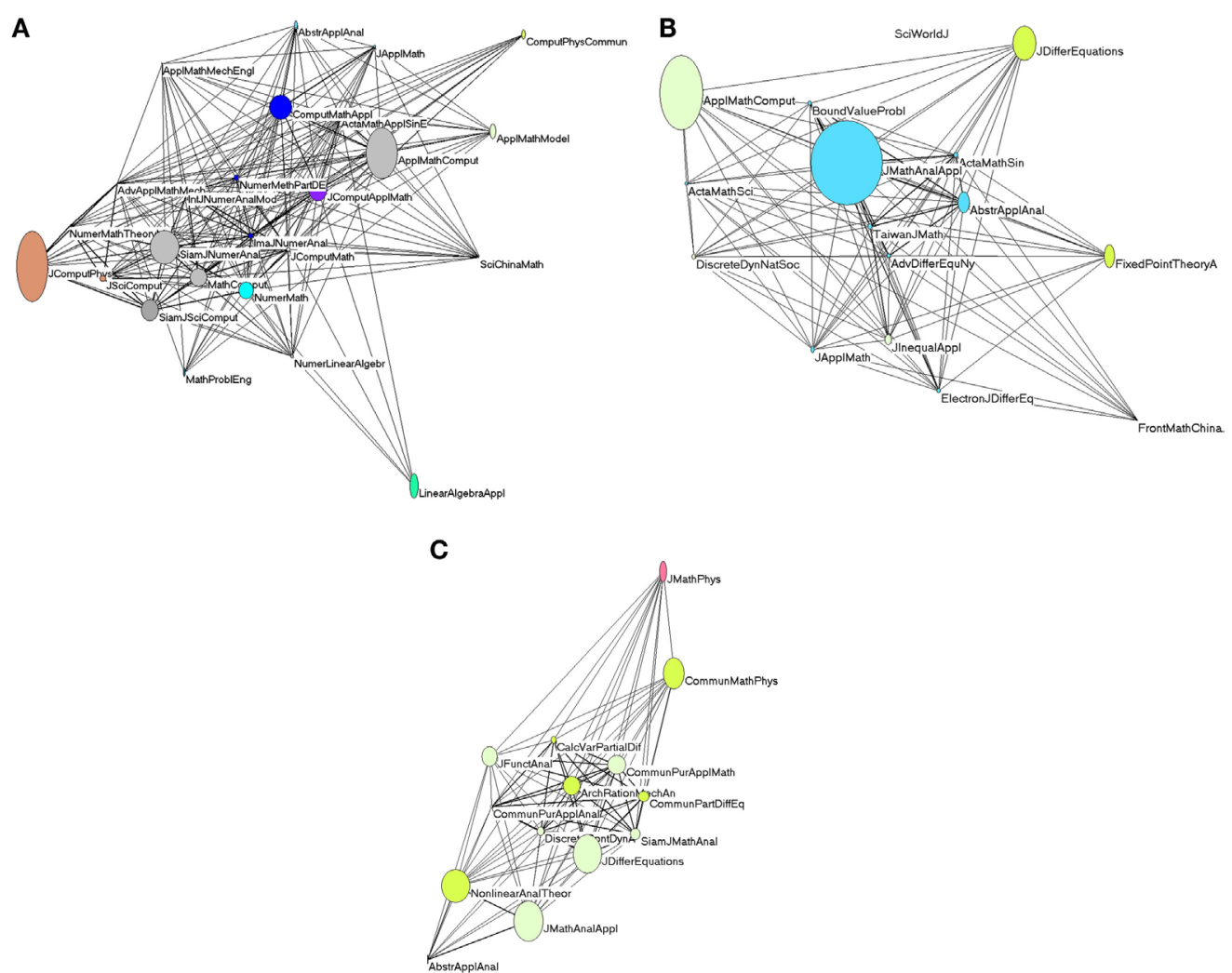

FIGURE 3 | Cited patterns of journals in mathematics in 2013 (threshold $=1 \%$, cosine $\geq 0.2$ ). (A) Journal of Computational Mathematics (JCM), (B) Acta Mathematica Scientia (AMS), and (C) Communications on Pure and Applies Mathematics (CPAM).

Differential Equations, are partly isolated from the network. With smallest node size in their cited networks, both JCM and AMS receive much fewer citations than journals with large node size. In other words, the overall citation impact (i.e., total citations in all years) of the two Chinese journals is very low compared with those in their cited network, such as Journal of Computational Physics, Applied Mathematics and Computation, Journal of Mathematical Analysis and Application, and Applied Mathematics and Computation.

Undoubtedly, the JIF value (3.080 in 2013) of the selected international journal - CPAM is significantly higher than those of the two Chinese journals, although decreased by $19.1 \%$ from that in 2008 (3.806) when there was no significant change in the number of citable items (respectively, 53 in 2008 and 48 in 2013). The JIF difference between CPAM and the two Chinese journals (JCM and AMS) is wide though narrowed from 3.041 in 2008 to 2.031 in 2013, respectively.

With 14 journals contributing to at least $1 \%$ of its total citations (Figure 3C), CPAM has wide-scope impact. The JIF values of journals in the cited network of CPAM range from 0.708 of CPAM to 3.080 of CPAM itself - CPAM outperforms the two Chinese journals in this regard. Journals in the cited network of CPAM are well integrated with each other, including those at the edge of the network. Surrounded by other journals in its cited network, CPAM has recognition in mathematics.

\section{DISCUSSION AND CONCLUSION}

We compared the citation impacts of Chinese journals supported or not supported by the CIUP so as to assess the selection rationality of the Plan, namely, whether supported journals perform better than those not supported, and those receive more financial support (i.e., rank higher with the CIUP) perform better than those receiving less. With limited options of journal samples defined by the CIUP, two sets of journals in environmental sciences and mathematics were selected. The results show that journals included in the CIUP perform better than those not included.

Journal impact factor values of the two Chinese journals in the environmental sciences (JESC supported by CIUP and BES not supported by CIUP) have increased from 2008 to 2013. JESC outperforms BES in overall citation impact, growth of absolute or relative ratio of JIF value, scope of citation impact in terms of the number of journals citing JESC, as well as JIF values of journals in JESC's cited network. JIF growth of JESC is higher than that of BES. With significantly more foreign journals and surrounded by journals in the same field, JESC's citation impact is wider and holes in its cited network are fewer. JESC is better integrated in the scholarly community in the environmental sciences.

Chinese journals in mathematics display another picture. Similar to the situation in the environmental sciences, CIUP 
supported journal (i.e., JCM) outperforms the one not supported (i.e., AMS) in terms of absolute JIF values. Nevertheless, AMS has progressed slightly faster in raising JIF value, and thus narrowing the gap with JCM. Both journals have similar impact in a journal set of similar JIF value scope and with high international visibilities. Compared with AMS, JCM is better integrated in the scholarly community and with fewer holes in its cited network.

In reducing gaps between Chinese journals indexed in the SCIE in terms of JIF values and structure of citation networks, those in mathematics perform better than those in the environmental sciences. Nevertheless, the gaps between Chinese and leading international journals are still wide in JIF value, the structure of the citation network, and overall citation impact. In general, it seems not an easy task to realize the goals set by the CIUP.

Financial support does help in infrastructure establishment or improvement as well as attracting talents to improve capacity of an editorial board. Money, however, is not almighty. In the Internet era, journal impact depends highly on paper quality and online accessibility. The most important issue to journal impact is quality of publications, expertise and dedication of editorial board members, editors, and reviewers. In the current administrative context of Chinese journals, it is common that editorial boards are mainly composed of administrative officials. Some members do have high academic honors but do not perform duties. Having an administrative title, regardless of one's educational background, is sometimes a condition for being included in a journal board. Furthermore, chief editors of many Chinese journals do not always have $\mathrm{PhD}$ degrees, let alone editors. For journals operationalized in this way, money can do little, if nothing, in raising citation impact.

In addition to intrinsic management challenges, the current research evaluation schemes in Chinese academic institutions put most Chinese journals in a disadvantageous situation: only publications in journals indexed by the Web of Science (WoS) are included in performance evaluation. Some leading Chinese universities assess papers based on journal Quartiles in JCR: a

\section{REFERENCES}

Davis, P. M., Lewenstein, B. V., Simon, D. H., Booth, J. G., and Connolly, M. J. (2008). Open access publishing, article downloads, and citations: randomized controlled trial. BMJ 337, a568. doi:10.1136/bmj.a568

Finardi, U. (2014). On the time evolution of received citations, in different scientific fields: an empirical study. J. Inform. 8, 13-14. doi:10.1016/j.joi.2013. 10.003

Gargouri, Y., Hajjem, C., Larivière, V., Gingras, Y., Carr, L., Brody, T., et al. (2010). Self-selected or mandated, open access increases citation impact for higher quality research. PLoS ONE 5:e13636. doi:10.1371/journal.pone.0013636

Harnad, S., and Brody, T. (2004). Comparing the impact of open access (OA) vs. non-OA articles in the same journals. Dlib Mag. 10, 6. Available at: http://www. dlib.org/dlib/june04/harnad/06harnad.html

He, C., and Pao, M. L. (1986). A discipline-specific journal selection algorithm. Inf. Process. Manage. 22, 405-416. doi:10.1016/0306-4573(86)90075-0

ISTIC. (2013). 中国科技论文统计结果 2013 (Statistical Data of Chinese SeT Papers). Beijing: Institute of Scientific and Technical Information of China.

Jin, B.H., and Rousseau, R. (2004). Evaluation of research performance and scientometric indicators in China. Handbook of quantitative science and technology paper in a higher quartile is scored higher. With so few Chinese journals being indexed in the WoS and most of them having low JIF values, attracting high-quality papers of Chinese authors is still a hard task for Chinese journals, let alone attracting highquality papers of international authors.

Visualization of the citation network helps assess journal impact in terms of integration into a scholarly community and overall citation impact (cited counts) or citation contribution (citing counts). Holes in a network most likely happen to multidisciplinary journals because of variable citation relations with journals of different fields: citations to multidisciplinary journals rely on citation practices in different fields. Low-impact journals may be less frequently read and thus difficult to receive sufficient attention for citations. The Chinese government may consider making all Chinese journals Open Access. This policy may be more effective than trying to change the behavior of authors, editors, and universities. As we have argued, accessibility is a precondition for being read and then cited (Harnad and Brody, 2004; Davis et al., 2008; Gargouri et al., 2010). A move to Open Access of Chinese journals across the board may change publication and marketing strategies of all parties involved.

\section{AUTHOR CONTRIBUTIONS}

PZ writes the paper and does most of the analysis. LL provides routines for harvesting journal citation networks, as well as comments and revision suggestions for the paper.

\section{ACKNOWLEDGMENTS}

The first author is supported by the National Natural Science Foundation of China (NSFC) with Grant Number 71473219 and the Planning Office of Philosophy and Social Sciences of Hangzhou City, Zhejiang Province, with Grant Number B14TD02. We thank Xianzan Lv and Xiaojing Cai for downloading data. We are grateful to Thomson Reuters for access to the JCR data. research, In: H. F. Moed, W. Glanzel, and U. Schmoch (eds). (Dordrecht: Kluwer Academic), 497-514.

Jin, B. H., Leydesdorff, L., Sun, H. R., Zhang, W., and Cen, Z. B. (2005). 中国科技期刊引文网络: 国际影响和国内影响分析 (Citation networks of Chinese S\&T journals: analysis on international and domestic impact). 中国科技期刊研究 (Chinese Journal of Scientific and Technical Periodicals) $16,141-146$.

Leydesdorff, L. (1986). The development of frames of references. Scientometrics 9, 103-125. doi:10.1007/BF02017235

Leydesdorff, L. (2007). Visualization of the citation environments of scientific journals: an online mapping exercise. J. Am. Soc. Inf. Sci. Technol. 58, 25-38. doi:10.1002/asi.20406

Leydesdorff, L., and Cozzens, S. E. (1993). The delineation of specialties in terms of journals using the dynamic journal set of the science citation index. Scientometrics 26, 133-154. doi:10.1007/BF02016797

Leydesdorff, L., and Jin, B. H. (2005). Mapping the Chinese science citation database in terms of aggregated journal-journal citation relations. J. Am. Soc. Inf. Sci. Technol. 56, 1469-1479. doi:10.1002/asi.20209

Li, Z. X. (2006). How to establish a first-class international scientific journal in China? World J. Gastroenterol. 12, 6905-6908. doi:10.3748/wjg.v12.i43.6905 
Liu, L. (2012). 解读“科技期刊国际影响力提升计划” (Understanding the international impact upgrading plan for journals in science and technology). 科技日报 (Science and Technology Daily) 3.

MOST. (2012). China Science \& Technology Statistics Database. Available at: http:// www.sts.org.cn/sjkl/kjtjdt/index.htm

NBS. (2013). "Expenditure on R\&D (100 million Yuan)," in China Statistical Year Book. Ren, S. L. (2005). Editing scientific journals in Mainland China. Eur. Sci. Edit. 31, 8-9. Available at: http://data.stats.gov.cn/english/easyquery.htm? $\mathrm{cn}=\mathrm{C} 01$

Salton, G., and McGill, M. J. (1983). Introduction to Modern Information Retrieval. Auckland: McGraw-Hill.

Slyder, J. B., Stein, B. R., Sams, B. S., Walker, D. M., Beale, B. J., Feldhaus, J. J., et al. (2011). Citation pattern and lifespan: a comparison of discipline, institution, and individual. Scientometrics 89, 955-966. doi:10.1007/s11192-0110467-x

Yao, Z. C., Luo, Z. F., Jin, X. Y., and Duan, R. Y. (2014). 新起点·新任务·新发展*《中国科技期刊国际影响力提升计划》资助期刊的分析与展望 (New start, new task, new development: analysis and expectation on journals funded by the International Impact Upgrading Plan for China’s S\&T journals) (in Chinese). 编辑学报 (Acta Editologica) 26, 342-346.

Zhou, P., and Leydesdorff, L. (2007a). A comparison between the china scientific and technical papers and citations database and the science citation index in terms of journal hierarchies and inter-journal citation relations. J. Am. Soc. Inf. Sci. Technol. 58, 223-236. doi:10.1002/asi.20475

Zhou, P., and Leydesdorff, L. (2007b). The citation impacts and citation networks of Chinese journals in mathematics. Scientometrics 72, 185-200. doi:10.1007/ s11192-007-1713-0

Zhou, P., and Leydesdorff, L. (2008). China ranks second in scientific publications since 2006. ISSI Newslett. 4, 7-9.

Zhou, P., Xinning, S., and Leydesdorff, L. (2010). A comparative study on communication structures of Chinese journals in the social sciences. J. Am. Soc. Inf. Sci. Technol. 61, 1360-1376. doi:10.1002/asi.21343

Conflict of Interest Statement: The authors declare that the research was conducted in the absence of any commercial or financial relationships that could be construed as a potential conflict of interest.

Copyright (C) 2016 Zhou and Leydesdorff. This is an open-access article distributed under the terms of the Creative Commons Attribution License (CC BY). The use, distribution or reproduction in other forums is permitted, provided the original author(s) or licensor are credited and that the original publication in this journal is cited, in accordance with accepted academic practice. No use, distribution or reproduction is permitted which does not comply with these terms. 